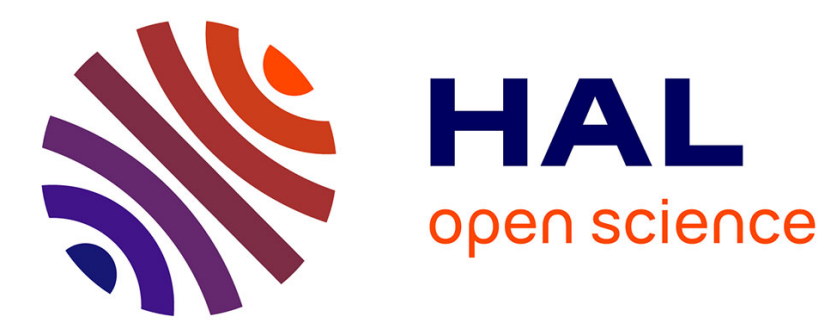

\title{
Application of the Fenchel Theorem to the Obstacle Problem
}

\author{
Diana R. Merluşcă
}

\section{To cite this version:}

Diana R. Merluşcă. Application of the Fenchel Theorem to the Obstacle Problem. 26th Conference on System Modeling and Optimization (CSMO), Sep 2013, Klagenfurt, Austria. pp.181-188, 10.1007/978-3-662-45504-3_17. hal-01286412

\section{HAL Id: hal-01286412 \\ https://hal.inria.fr/hal-01286412}

Submitted on 10 Mar 2016

HAL is a multi-disciplinary open access archive for the deposit and dissemination of scientific research documents, whether they are published or not. The documents may come from teaching and research institutions in France or abroad, or from public or private research centers.
L'archive ouverte pluridisciplinaire HAL, est destinée au dépôt et à la diffusion de documents scientifiques de niveau recherche, publiés ou non, émanant des établissements d'enseignement et de recherche français ou étrangers, des laboratoires publics ou privés. 


\title{
Application of the Fenchel theorem to the obstacle problem ${ }^{\star}$
}

\author{
Diana R. Merluşcă \\ Institute of Mathematics of the Romanian Academy \\ Bucharest, Romania \\ dianam1985@yahoo.com
}

\begin{abstract}
In this paper we apply a duality algorithm to the general obstacle problem for second order operators. We reduce the problem to the null obstacle case and we solve it by using an algorithm based on a dual approximate problem. This method generates a quadratic minimization problem, which is easy to implement numerically. The convergence properties and the numerical results show that the algorithm is working properly for any admissible obstacle.
\end{abstract}

Keywords: obstacle problem, duality, approximate problem.

\section{Introduction}

The obstacle problem is a very well studied subject. Many methods have been applied for solving it. For instance, Glowinski 6 used the finite element method for solving the null obstacle problem, while Barbu and Precupanu [2] studied the problem from the duality point of view. The general obstacle problem is also intensively studied for its wide range of applications in mechanics and physics. We refer here to Rodrigues [14, Caffarelli and Friedman [4], Duvaut and Lions [5] and Ciarlet 3 .

We extend here the the duality method developed in the articles Merluşcă [8, 9], by the application of the Fenchel theorem to the obstacle problem. We discuss the general obstacle problem in Section 2. We reduce the problem to the null obstacle case and we compute the solutions using the duality method (Merluşcă [9]). In Merluşcă [10, the case of the fourth order obstacle problem was analysed. In section 3, we apply this technique in numerical examples for one dimensional problems and the obtained results are very accurate. Finally, we mention the works of Neittaanmaki, Sprekels and Tiba 12 and Sprekels and Tiba [15], where a related duality approach was used in the study of KirchhoffLove arches and explicit solutions were obtained.

\footnotetext{
^ This paper is supported by the Sectorial Operational Programme Human Resources Development (SOP HRD), financed from the European Social Fund and by the Romanian Government under contract number SOP HRD/107/1.5/S/82514
} 


\section{The general obstacle problem for second order equations}

We consider the following obstacle problem

$$
\min \left\{\frac{1}{2} \int_{\Omega}|\nabla y|^{2}-\int_{\Omega} f y: y \in K_{\psi}\right\}
$$

where $K_{\psi}=\left\{y \in H_{0}^{1}(\Omega): y \geq \psi\right\}, \psi \in H^{1}(\Omega)$ is such that $\left.\psi\right|_{\partial \Omega} \leq 0$ and $f \in L^{2}(\Omega)$.

It is known that the unique solution of problem (1) is an element in $H^{2}(\Omega)$ (Th. 2.5, 1]).

Lemma 1. Let $y_{\psi}$ be the solution of the problem (1) and $\hat{y}$ the solution of the problem

$$
\begin{gathered}
-\Delta \hat{y}=f, \text { on } \Omega, \\
\hat{y}=0, \quad \text { on } \partial \Omega,
\end{gathered}
$$

then $y_{\psi} \geq \hat{y}$ almost everywhere on $\Omega$.

The problem (1) in which we replace $\psi$ by $\hat{\psi}=\max \{\hat{y}, \psi\} \in H_{0}^{1}(\Omega)$ has the same solution $y_{\psi}$.

Proof. Denoting $\beta \subset \mathbb{R} \times \mathbb{R}$ a maximal monotone operator defined by

$$
\beta(z)= \begin{cases}]-\infty, 0], & z=0, \\ 0, & z>0, \\ \emptyset, & z<0 .\end{cases}
$$

we rewrite (1) as

$$
-\Delta y_{\psi}+\beta\left(y_{\psi}-\psi\right) \ni f \text { in } \Omega .
$$

Then, since $y_{\psi} \in H^{2}(\Omega), \beta\left(y_{\psi}-\psi\right) \in L^{2}(\Omega)$ and $\beta\left(y_{\psi}-\psi\right) \leq 0$ a.e. on $\Omega$. By a comparison of $(2)$ and $(3)$, we obtain that $y_{\psi} \geq \hat{y}$ a. e. on $\Omega$.

We denote $\hat{K}=\left\{y \in H_{0}^{1}(\Omega): y \geq \hat{\psi}\right\}$. Then $y_{\psi} \in \hat{K}, \Delta y_{\psi}+f \leq 0$ a.e. on $\Omega$.

For every $v \in \hat{K}$, we compute

$$
\begin{aligned}
\int_{\Omega}\left(\Delta y_{\psi}+f\right)\left(v-y_{\psi}\right) & =\int_{\Omega}\left(\Delta y_{\psi}+f\right)\left(\hat{\psi}-y_{\psi}\right)+\int_{\Omega}\left(\Delta y_{\psi}+f\right)(v-\hat{\psi}) \\
& \leq \int_{\Omega}\left(\Delta y_{\psi}+f\right)\left(\hat{\psi}-y_{\psi}\right)=0 .
\end{aligned}
$$

The last equality is due to the classical formulation of the obstacle problem (the complementarity property)

$$
\begin{aligned}
-\Delta y_{\psi} & =f, \quad \text { in } \Omega^{+}=\left\{y_{\psi} \in \Omega: y_{\psi}(x)>\psi(x)\right\}, \\
-\Delta y_{\psi} & \geq f, \quad \text { in } \Omega \backslash \Omega^{+}=\left\{y_{\psi} \in \Omega: y_{\psi}(x)=\psi(x)\right\}, \\
y_{\psi} & =\psi \text { and } \frac{\partial y_{\psi}}{\partial n}=\frac{\partial \psi}{\partial n}, \quad \text { on } \partial \Omega^{+} \cap \Omega, \\
y_{\psi} & =0 \quad \text { on } \partial \Omega .
\end{aligned}
$$


and to the fact that $y_{\psi}(x)=\psi(x)$ means that $\hat{y}(x) \leq \psi(x)$ and that yields that $y_{\psi}(x)=\hat{\psi}(x)$.

Then integrating by parts we get

$$
\int_{\Omega} \nabla y_{\psi} \nabla\left(v-y_{\psi}\right) \leq \int_{\Omega} f\left(v-y_{\psi}\right), \quad \forall v \geq \hat{\psi} .
$$

Remark 1. Lemma 1 is known (see Murea and Tiba [1]) and we indicate its proof for easy reference.

The null obstacle problem that we use is

$$
\min _{y \in K_{0}}\left\{\frac{1}{2} \int_{\Omega}|\nabla y|^{2}-\int_{\Omega} f y+\int_{\Omega} \nabla \hat{\psi} \nabla y\right\} .
$$

Let $y_{0}$ be the unique solution of problem (4).

Proposition 1. Then the solution of the problem (1) can be computed by just adding $\hat{\psi}$, i.e.

$$
y_{\psi}=y_{0}+\hat{\psi} .
$$

Proof. The weak formulation of problem (4) is given by the form

$$
\int_{\Omega} \nabla y_{0} \nabla\left(y_{0}-v\right) \leq \int_{\Omega} f\left(y_{0}-v\right)-\int_{\Omega} \nabla \hat{\psi} \nabla\left(y_{0}-v\right), \quad \forall v \in K_{0} .
$$

We translate the problem by adding $\hat{\psi}$. Then, for every $v \in K_{0}$, we get $v+\hat{\psi} \geq$ $\hat{\psi} \geq \psi$. With this translations from the variational inequality we obtain

$$
\int_{\Omega} \nabla\left(y_{0}+\hat{\psi}\right)\left(\nabla\left(y_{0}+\hat{\psi}\right)-\nabla(\hat{\psi}+v)\right) \leq \int_{\Omega} f\left(y_{0}+\hat{\psi}-v-\hat{\psi}\right) .
$$

Using Lemma 1 it yields that, by a translation with $\hat{\psi}$, the problem (4) is equivalent to problem (1). Then we conclude that $y_{0}+\hat{\psi}=y_{\psi}$.

Since $\int_{\Omega} \nabla y \nabla \hat{\psi}=-\int_{\Omega} \Delta \hat{\psi} y$ and $\Delta \hat{\psi} \in H^{-1}(\Omega)$, then we can consider the approximate problem

$$
\min \left\{\frac{1}{2} \int_{\Omega}|\nabla y|^{2}-\int_{\Omega}(f+\Delta \hat{\psi}) y: y \in C_{k}\right\}
$$

where $C_{k}=\left\{y \in H_{0}^{1}(\Omega): y\left(x_{i}\right) \geq 0, \forall i=1,2, \ldots, k\right\}$ and $\left\{x_{i}\right\}_{i}$ is a dense set in $\Omega$.

In (6), we assume that $\operatorname{dim} \Omega=1$ (for the numerical applications in Section 3 ), but the result can be extended in higher dimension by using non hilbertian Sobolev spaces. Using the Sobolev imbedding theorem and the weak lower semicontinuity of the norm, then we can prove the following approximation result (see Merluşcă 9]) 
Theorem 1. The sequence $\left\{\bar{y}_{k}\right\}_{k}$ of the solutions of problems $(6)$, for $k \in \mathbb{N}$, is a strongly convergent sequence in $H_{0}^{1}(\Omega)$ to the unique solution $\bar{y}$ of the problem (4).

We denote $\hat{f}=f+\Delta \hat{\psi} \in H^{-1}(\Omega)$. Applying the Fenchel duality theorem to problem (6) we obtain the dual problem

$$
\min \left\{\frac{1}{2}\left|y^{*}+\hat{f}\right|_{H^{-1}(\Omega)}^{2} \quad: y \in C_{k}^{*}\right\}
$$

where $C_{k}^{*}=\left\{y^{*} \in H^{-1}(\Omega): y^{*}=\sum_{i=1}^{k} \alpha_{i} \delta_{x_{i}}, \alpha_{i} \geq 0\right\}$ is the dual cone.

Remark 2. Let $y_{k}^{*}$ be the solution of the dual approximate problem (7). Since $y_{k}^{*} \in C_{k}^{*}$, it is sufficient to compute the coefficients $\alpha_{i}^{*}$, due to the formula

$$
y_{k}^{*}=\sum_{i=1}^{k} \alpha_{i}^{*} \delta_{x_{i}} .
$$

The solution $y_{k}$ of the approximate problem (6) is computed using the equality $y_{k}=J^{-1}\left(y_{k}^{*}+\hat{f}\right)$, where $J$ is the duality mapping $J: H_{0}^{1}(\Omega) \rightarrow H^{-1}(\Omega)$ and we also have $\alpha_{i}^{*} y_{k}\left(x_{i}\right)=0, \quad \forall i=\overline{1, k}$.

We obtain the formula for the solution of the approximate problem, denoted by $y_{k}^{0}$,

$$
y_{k}^{0}=\sum_{i=1}^{k} \alpha_{i}^{*} J^{-1}\left(\delta_{i}\right)+J^{-1}(\hat{f})
$$

using the fact that the duality mapping $J: H_{0}^{1}(\Omega) \rightarrow H^{-1}(\Omega)$ is defined by $J(y)=-\Delta y$.

Then applying (5) we find the approximate solution of the general obstacle problem (1).

\section{$3 \quad$ Numerical applications}

In this section we discuss two examples in one dimension for the general obstacle problem for second order operators.

We consider the obstacle problem (1) with $\Omega=]-1,1[$ the domain, $\psi \equiv-1 / 18$ the obstacle and

$$
f(x)= \begin{cases}-1, & |x|>1 / 4 \\ 1-32 x^{2}, & |x| \leq 1 / 4\end{cases}
$$

The solution of this problem is, Ockendon and Elliott [13, (pp. 93 - 94)

$$
u(x)= \begin{cases}-\frac{1}{18}+\frac{1}{2}\left(x \pm \frac{2}{3}\right)^{2}, & \frac{2}{3}<|x| \leq 1, \\ -\frac{1}{18}, & \frac{1}{3} \leq|x| \leq \frac{2}{3}, \\ -\frac{1}{18}+\frac{1}{2}\left(x \pm \frac{1}{3}\right)^{2}, & \frac{1}{4} \leq|x|<\frac{1}{3}, \\ -\frac{1}{32}+\frac{8}{3} x^{2}\left(x^{2}-\frac{3}{16}\right), & |x|<\frac{1}{4}\end{cases}
$$


The duality mapping $J: H_{0}^{1}(\Omega) \rightarrow H^{-1}(\Omega)$ is defined as $J(y)=-y^{\prime \prime}$. It is a linear bounded operator.

We consider the discretization $x_{i}=2 i h-1$, where $h=1 / k$, for all $i \in$ $\{0,1,2, \ldots, k\}$.

We denote by $d_{i}=J^{-1}\left(\delta_{x_{i}}\right)$. Computing them we get

$$
d_{i}(x)=\left\{\begin{array}{lr}
0.5\left(1-x_{i}\right)(x+1), & x \leq x_{i}, \\
0.5\left(1-x_{i}\right)(x+1)-x+x_{i}, x>x_{i},
\end{array} \quad \forall i \in\{0,1, \ldots, k\} .\right.
$$

We consider $y_{f}=J^{-1}(f+\Delta \hat{\psi})$ the solution of the problem

$$
\begin{aligned}
& \left.-y_{f}^{\prime \prime}=f+\Delta \hat{\psi}, \quad \text { on }\right]-1,1[, \\
& y_{f}(-1)=y_{f}(1)=0 .
\end{aligned}
$$

Then

$$
\left|y^{*}+f+\Delta \hat{\psi}\right|_{H^{-1}(\Omega)}^{2}=\left|\sum_{i=1}^{k} \alpha_{i} d_{i}+y_{f}\right|_{H_{0}^{1}(\Omega)}^{2}
$$

and

$$
\left|\sum_{i=1}^{k} \alpha_{i} d_{i}+y_{f}\right|_{H_{0}^{1}(\Omega)}^{2}=\sum_{i, j=1}^{k} \alpha_{i} \alpha_{j} \int_{\Omega} d_{i}^{\prime} d_{j}^{\prime}+2 \sum_{i=1}^{k} \alpha_{i} \int_{\Omega} d_{i}^{\prime} y_{f}^{\prime}+\int_{\Omega}\left(y_{f}^{\prime}\right)^{2} .
$$

Denoting $a_{i j}=\int_{\Omega} d_{i}^{\prime} d_{j}^{\prime}, b_{i}=\int_{\Omega} d_{i}^{\prime} y_{f}^{\prime}$, we solve the dual problem (7) which is equivalent to the quadratic minimization problem

$$
\min _{\alpha \geq 0} \frac{1}{2} \alpha^{T} A \alpha+b^{T} \alpha,
$$

where $A=\left[a_{i j}\right]$ and $b=\left[b_{i}\right]$

Computing the components, we get $b_{i}=y_{f}\left(x_{i}\right)$ and

$$
a_{i j}=\left\{\begin{array}{l}
0.5\left(1+x_{i}\right)\left(1-x_{j}\right), j>i \\
0.5\left(1+x_{j}\right)\left(1-x_{i}\right), j \leq i
\end{array}\right.
$$

In Figure 1 we represent the coefficients $\left\{\alpha_{i}^{*}\right\}_{i=1,100}$, the solution of the problem (8).

We construct the solution of the problem (6) using Remark 2 then we apply formula (5) to obtain the approximate solution of (1).

In Figure 2 we represent three solutions: the one computed by the duality method, the one computed with the IPOPT optimizer (Freefem++ script included in the ff-Ipopt dynamic library, Hatch [7]; for details about the method see Wächter and Biegler [16]) and the exact solution given by Ockendon and Elliot 13. They coincide graphically.

We now consider an example with general obstacle:

$$
\min \left\{\frac{1}{2} \int_{\Omega}|\nabla y|^{2}-\int_{\Omega} f y: y \in K_{\psi}\right\}
$$




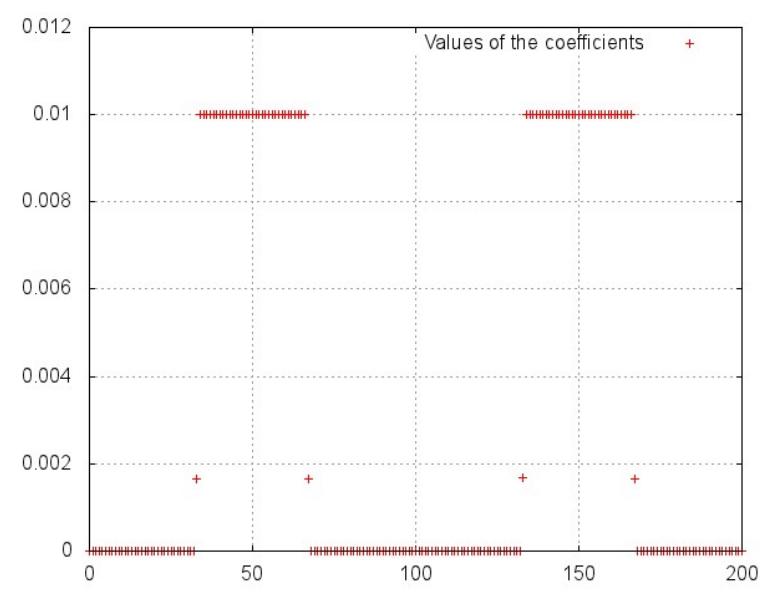

Fig. 1: The coefficients $\left\{\alpha_{i}^{*}\right\}_{i}$.

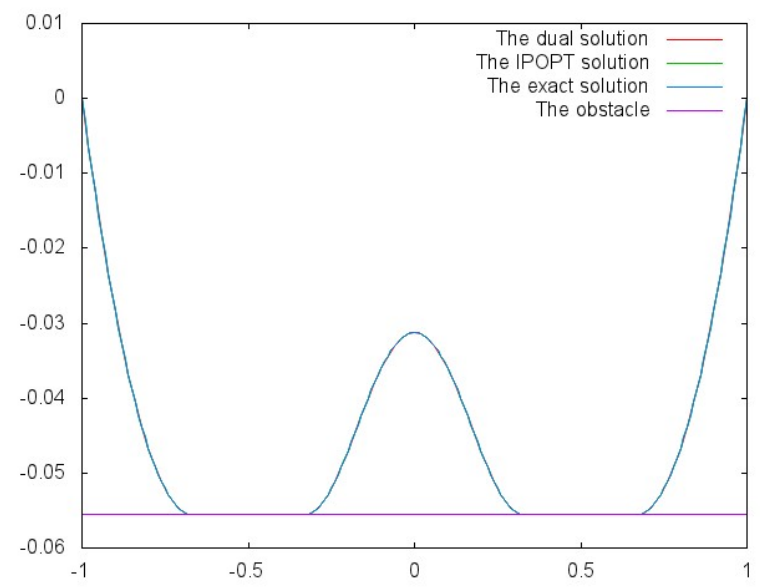

Fig. 2: The exact solution, the dual solution and the IPOPT solution are graphically identical. 
where $\left.K_{\psi}=\left\{y \in H_{0}^{1}(\Omega): y \geq \psi\right\}, \Omega=\right]-1,1\left[, \psi(x)=-x^{2}+0.5\right.$ and

$$
f(x)= \begin{cases}-10, & |x|>1 / 4 \\ 10-x^{2}, & |x| \leq 1 / 4\end{cases}
$$

After solving the quadratic minimization problem (8), the solution of which is represented in Figure 3, we compute the solution of the approximate problem (6) using the Remark 2

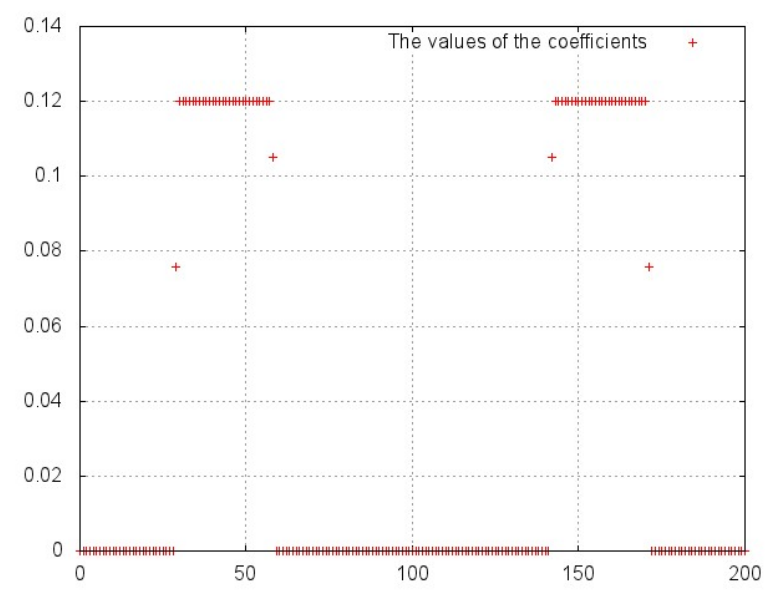

Fig. 3: The coefficients $\left\{\alpha_{i}^{*}\right\}_{i}$.

We represent in Figure 4 the obstacle $\psi$ and the solutions, one computed by the duality method and the other one computed by the IPOPT method [7]. The two solutions are very close and coincide graphically.

\section{References}

1. Barbu, V.: Optimal control of variational inequalities. Research Notes in Mathematics, 100. Pitman, Boston (1984)

2. Barbu V.: Precupanu T. Convexity and optimization in Banach spaces, Noordhoff (1978)

3. Ciarlet P.G.: Numerical analysis of the finite element method, Les Presses de l'Universitè de Montrèal (1976)

4. Caffarelli, L. A., Friedman, A.: The obstacle problem for the biharmonic operator. Annali della Scuola Normale Superiore di Pisa-Classe di Scienze, 6(1), 151-184 (1979)

5. Duvaut, G., Lions, J.-L.: Les inéquations en mécanique et en physique. Travaux et Recherches Mathematiques, N. 21. Dunod, Paris (1972)

6. Glowinski, R.: Numerical Methods for Nonlinear Variational Problems, SpringerVerlag, New York (1984) 


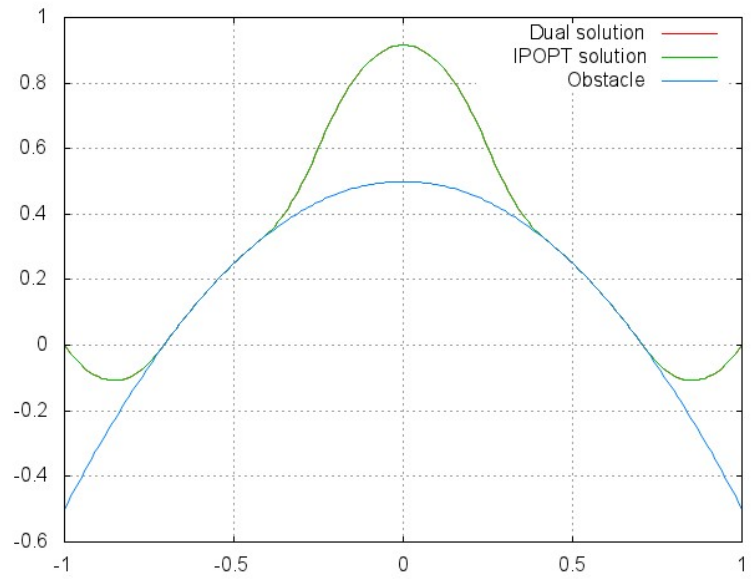

Fig. 4: The dual solution and the IPOPT solution coincide graphically.

7. Hatch, F.: Freefem documetation, Laboratoire Jacques-Louis Lions, Université Pierre et Marie Curie, Paris (2013) http://www.freefem.org/ff+t/ftp/freefem+ + doc.pdf

8. Merluşcă, D.R.: A duality algorithm for the obstacle problem. Annals of the Academy of Romanian Scientists, Vol. 5, No. 1-2, 209-215 (2013)

9. Merluşcă, D. R.: A duality-type method for the obstacle problem. Analele Stiintifice ale Universitatii Ovidius Constanta, Seria Matematica, Vol. 21(3), pp. 181-195 (2013)

10. Merluşcă, D. R.: A Duality-Type Method For The Fourth Order Obstacle Problem, submitted to U.P.B. Sci. Bull., Series A.

11. Murea, C.M., Tiba, D.: A direct algorithm in some free boundary problems, BCAM Publications (2012) http://www.bcamath.org/documentos_public/ archivos/publicaciones/obstacle_2012-07-06.pdf

12. P.Neittaanmaki,Sprekels J., Tiba D.: "Optimization of elliptic systems. Theory and applications, Springer Monographs in Mathematics, Springer Verlag, New York (2006)

13. Ockendon, M. C., Elliot, J. R.: Weak and variational methods for moving boundary problems, Pitman Pub. edition (1982)

14. Rodrigues, J. F.: Obstacle problems in mathematical physics. Elsevier Science, North-Holland (1987)

15. Sprekels J., Tiba D.: Sur les arches lipschitziennes (French) C.R.A.S. Paris Ser.I Math. vol.331, no.2, p.179-184 (2000)

16. Wächter, A., Biegler, L. T.: On the Implementation of a Primal-Dual Interior Point Filter Line Search Algorithm for Large-Scale Nonlinear Programming, Mathematical Programming 106(1), pp. 25-57 (2006) 